\title{
Acute Changes in Autonomic Nerve Activity during Passive Static Stretching
}

\author{
Takayuki Inami ${ }^{1, *}$, Takuya Shimizu ${ }^{2}$, Reizo Baba ${ }^{3}$, Akemi Nakagaki ${ }^{4}$ \\ ${ }^{1}$ School of Exercise and Health Sciences, Edith Cowan University, Joondalup Drive, Joondalup, WA, Australia \\ ${ }^{2}$ Graduate School of Health and Sports Sciences, Chukyo University, Tokodachi, Toyota, Aichi, Japan \\ ${ }^{3}$ Department of Pediatric Cardiology, Aichi Children's Health and Medical Center, Osakada, Obu, Aichi, Japan \\ ${ }^{4}$ Reproductive Health Nursing/Midwifery, Graduate School of Nursing, Nagoya City University, Japan \\ *Corresponding author: inami0919@gmail.com
}

Received June 06, 2014; Revised July 12, 2014; Accepted July 16, 2014

\begin{abstract}
This study aimed to investigate the acute change of static stretching (SS) on autonomic nerve activity and to clarify the effect of SS on systemic circulation. Twenty healthy young, male volunteers performed a 1-min SS motion of the right triceps surae muscle, repeated five times. The autonomic nerve activity balance was obtained using second derivatives of the photoplethysmogram readings before (pre), during, and after (post) SS. Heart rate and blood pressure (BP) were also measured. The autonomic nerve activity significantly changed to parasympathetic dominance by SS as compared with pre. In addition, for SS, the autonomic nerve activity slowly changed to sympathetic dominance after completion of all sets of stretching, but these value did not return to pre during the 5 minutes after the completion of all sets of stretching, with parasympathetic dominance continuing by 4 minutes after SS. The BP and HR transiently increased during SS and decreased after SS. In addition, HR significantly decreased after completion of all sets of SS.The possibility that the response during SS may differ from the response during active static stretching is shown.
\end{abstract}

Keywords: sympathetic nerve activity, parasympathetic nerve activity, triceps surae muscle, static stretching, blood pressure, heart rate

Cite This Article: Takayuki Inami, Takuya Shimizu, Reizo Baba, and Akemi Nakagaki, "Acute Changes in Autonomic Nerve Activity during Passive Static Stretching." American Journal of Sports Science and Medicine, vol. 2, no. 4 (2014): 166-170. doi: 10.12691/ajssm-2-4-9.

\section{Introduction}

Static stretching (SS) is a form of physical exercise in which a specific skeletal muscle (or muscle group) is deliberately stretched and reflects the mechanical characteristics of skeletal muscle. It is widely used to increase articular range of motion (ROM) by favorably affecting the flexibility of muscles and tendons $[1,2]$. In addition, it is reported that SS can provide "relaxation" like the techniques employed in the field of psychology $[3,4]$.These reports have indicated that extension stimuli on the muscles may induce advantageous changes in the balance of autonomic nerve activity; however, only a small number of studies focusing on SS and autonomic nerve activity have been conducted.

We could find three studies in which SS was evaluated by analyzing the changes in autonomic nerve activity based on the changes in heart rate variability (HRV) in human subjects. Saito, et al. [5] conducted SS (trunk flexion) on healthy volunteers and showed that parasympathetic nerve activity was significantly higher after SS than it was before SS.Farinatti, et al. [6] applied SS (trunk flexion) to subjects with a low level of flexibility and showed that parasympathetic nerve activity decreased remarkably during SS and was significantly higher after SS than it was before SS. Mueck-Weymann, et al. [7] conducted SS on the large muscles of bodybuilding athletes for 28 days and confirmed a significant increase in parasympathetic nerve activity and a significant decrease in sympathetic nerve activity after the completion. Based on these findings, it can be understood that the balance of autonomic nerve activity shifts to the sympathetic nerve activity-dominant state during SS and the parasympathetic nerve activity-dominant state after SS. All of these reports involve the response to active SS; however, there is no report on autonomic nerve activity relating to passive SS. According to Mohr, et al. [8],SS has to be conducted first in order to achieve the maximum effect of SS, and Alter [2] stated that the tension caused by muscle contraction must be suppressed to the minimum in order to minimize active resistance. These precedent studies have a problem in that there is an extremely high possibility that the muscles other than the target muscle are under tension, because the trunk flexion was conducted actively. Actually, the influence of SS on the nervous system is known to be transmitted to the sites to which SS is not conducted $[9,10]$. It is thus assumed that active SS and passive SS have different influences on the autonomic nerve activity, although the term "SS" is used collectively. 
We hypothesizes that the changes in autonomic nerve activity upon passive SS are different from the response following active SS. This study aims at investigating the acute effect on autonomic nerve activity when SS is conducted passively.

\section{Materials and Methods}

\subsection{Participants}

This study was approved by the local ethics committee and conducted in accordance with the Declaration of Helsinki. The purpose, procedures, and risks of the study were informed, and a written informed consent was obtained from each participant. Twenty non-smoking, healthy male adults (aged 18 to 20 years, 19.3 years on average) without cardiovascular, orthopedic, or neurological diseases were recruited as study subjects. SS was conducted for the right triceps surae muscle. They had not been involved in any resistance training or stretching program before the study. The sample size was calculated on the basis of an $\alpha$ level of 0.05 and a power $(1-\beta)$ of 0.8 , with an estimated 20\% difference in ROM before and after of SS using data from a previous study [11]. Their height was $175.0 \pm 6.4 \mathrm{~cm}$ (mean \pm standard error: the same below) and body weight was $68.9 \pm 8.2 \mathrm{~kg}$.

\subsection{Study Design}

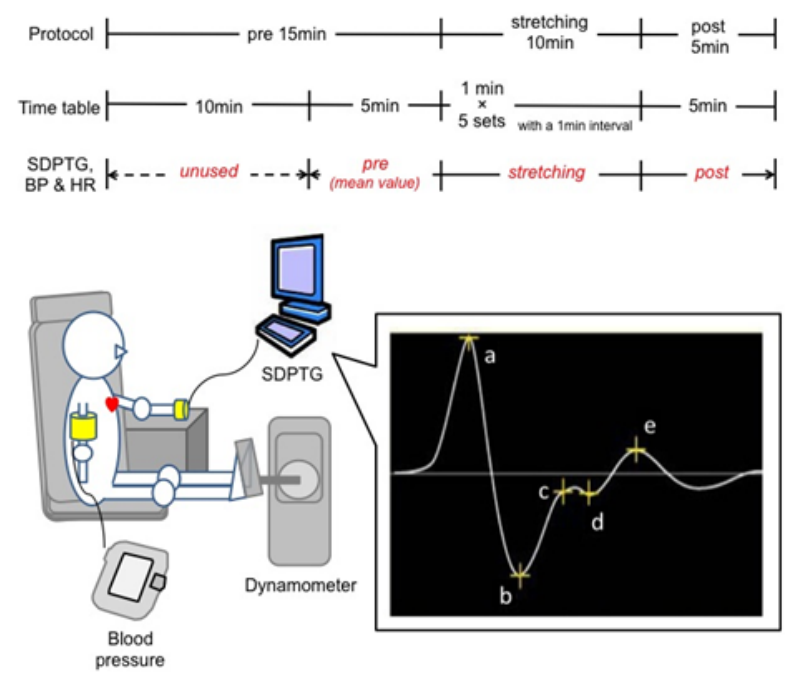

Figure 1. Protocol and measurement system

The study participants visited the laboratory on three occasions at the same time of day, with at least $48 \mathrm{~h}$ between visits; all experimental trials were completed within 3weeks. A full familiarization with the SS protocol and test procedures was provided during the first session, whereas the subsequent two visits were used to complete the following experimental protocol, in a randomized order: 1) control session (no stretching); 2) five sets of 1min passive plantar flexor SS, as described previously $[11,12]$. Data were collected during a period of 30-min including these stretching sessions, a period of resting in the sitting position (the knee fully extended) for 15-min before stretching (referred to as "pre" below), and a 5-min period after stretching (referred to as "post" below). The temperature in the experimental room was set at $25^{\circ} \mathrm{C}$. The subjects were asked not to consume any alcohol on the day before measurement and not eat breakfast on the day of measurement. The experiment was conducted while external environmental factors that could affect measurement were minimized, and care was taken to ensure subject silence and comfort. The protocol and measurement system are shown in Figure 1.

\subsection{Static Stretching Protocol}

Two techniques, SS and a control with no stretching were used, and SS was conducted passively to minimize active resistance. In SS, the knee joint was in the extended position and the ankle joint in the maximally dorsiflexed position in a sitting position (the knee fully extended) [13]. For control the subjects rested in sitting position (the knee fully extended). The load with which a subject himself felt to have an "appropriate stretched feeling" or "slightly taut feeling" [14] was measured in advance with a hand-held dynamometer (Loadcell LU-100KSB34D of Kyowa Electronic Instruments Co., Ltd.; Strain amplifier: F-420 of Uniplus Corporation), and the passive external force during repeated stretching was controlled to impose the same load in the respective sets of the respective stretching. This position was then held at a constant angle for $1 \mathrm{~min}$, and this stretching procedure was repeated 5 times with a 1-min interval between sets (total 10-min). In addition, the maximum ROM of the ankle joint was measured with a goniometer of Tokyo University [13].

\subsection{Measurement of Autonomic Nerve Activity, Blood Pressure (BP) and Heart Rate (HR)}

A large number of attempts of evaluating the balance of autonomic nerve activity by analyzing the waveform of an electrocardiogram and pulse wave have been reported as evaluation of autonomic nerve activity. The method involving frequency analysis of an electrocardiogram or second derivative of photoplethysmogram (SDPTG) quantifies sympathetic nerve activity and parasympathetic nerve activity separately and is clinically applied. In particular, SDPTG performs measurements using an optical sensor noninvasively at the fingertips and reflects changes in the absorbance of hemoglobin independently from skin tension or properties of the subcutaneous fat, and thus involves more noninvasive characteristics than electrocardiography in which electrodes are attached. The waveform of SDPTG is composed of five components, a through e. It is reported that the a-a interval of SDPTG and the R-R interval of electrocardiogram are highly correlated with a correlation coefficient of 0.992 from young to middle-aged to elderly individuals; this is reportedly higher than the 0.977 correlation coefficient between the R-R interval of electrocardiogram and finger photoplethysmogram [15]. Further, it is shown in the report that the spectrum power values obtained from the SDPTG a-a interval correspond to the spectrum power values obtained from electrocardiogram from the lowfrequency band to the high-frequency band [15]. Accordingly, analysis of autonomic nerve activity using SDPTG has physiological significance equivalent to that obtained using electrocardiogram.

A SDPTG (Artett C of U-Medica Inc., Osaka, Japan) was used for the measurement of autonomic nerve activity. The pulse waveforms (a to e waves: Figure 1) output on a personal computer were used for frequency analysis for 
the a-a interval using software for autonomic function evaluation and analysis exclusively used for Artett. Based on the results of the frequency analysis, the low-frequency component (LF) was set at 0.04 to $0.15 \mathrm{~Hz}$ and the high frequency component (HF) at 0.15 to $0.4 \mathrm{~Hz}$ [16]. The power spectral densities at the respective frequency zones were calculated, and the LF and HF (mainly parasympathetic nerve activity) and their ratio $\mathrm{LF} / \mathrm{HF}$ (mainly sympathetic nerve activity), and HR were obtained every 1-min. Since HF and LF/HF differ greatly among individuals, normalization was performed so that the means of $\mathrm{HF}$ and $\mathrm{LF} / \mathrm{HF}$ were 0 and the standard deviation was 1 during the continuous measurement period for each subject, and the HF and LF/HF converted into normal distribution are expressed as nHF and $n(\mathrm{LF} / \mathrm{HF})$, respectively. Because the balance of the autonomic nerve activity have a reciprocal relationship, in the estimation of the balance of the autonomic nerve activity, when

$$
\mathrm{nHF}-\mathrm{n}(\mathrm{LF} / \mathrm{HF})>0
$$

it was considered to be parasympathetic nerve activitydominant, and when

$$
\mathrm{nHF}-\mathrm{n}(\mathrm{LF} / \mathrm{HF})<0
$$

it was considered to be sympathetic nerve activitydominant [17].
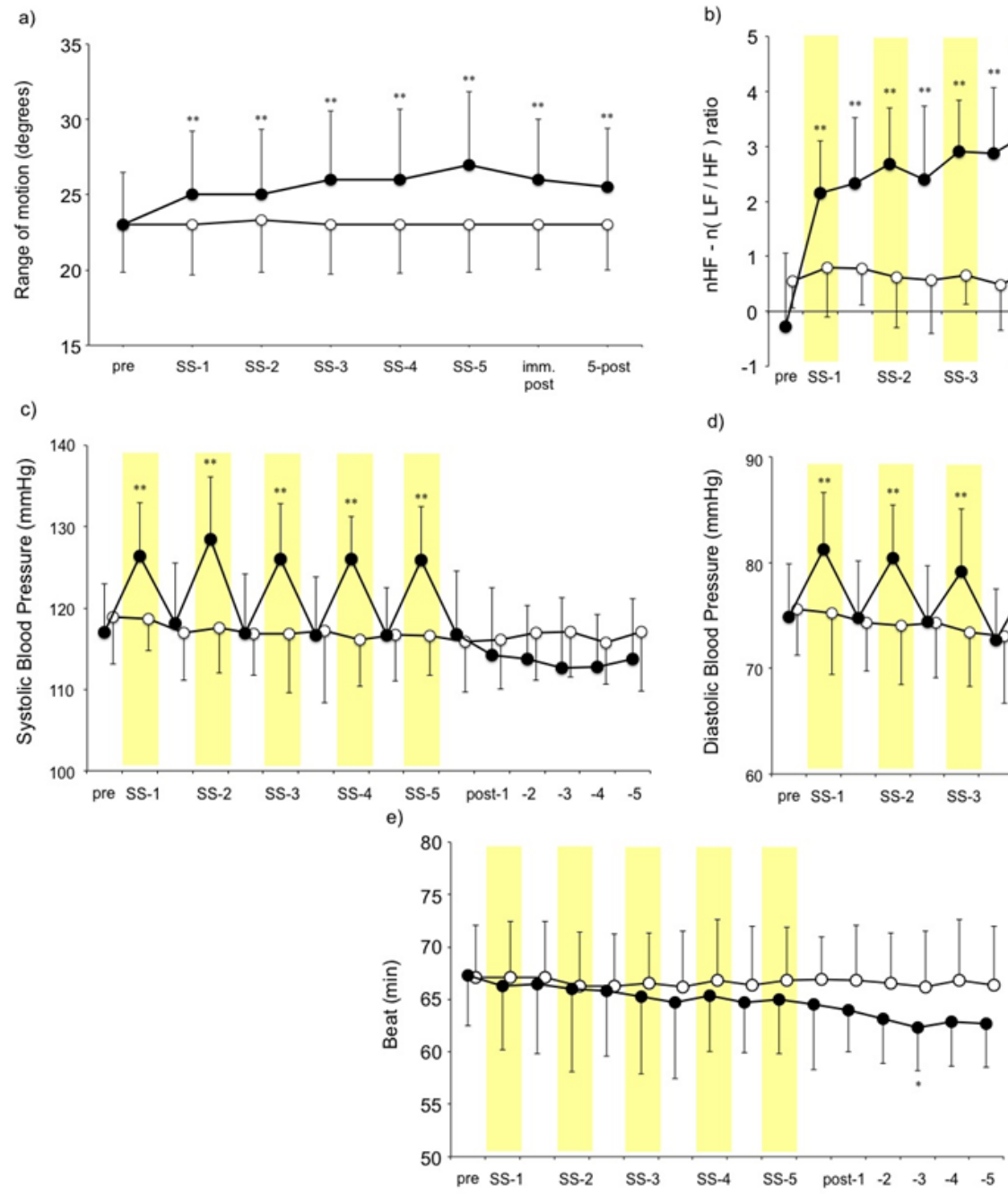

Figure 2. Changes in each parameter

It is shown that changes in a) ROM, b) autonomic nerve activity, c) SBP, d) DBP, and e) HR. The yellow makers indicate the SS phase. *: $\mathrm{p}<0.05 ; * *: \mathrm{p}<0.01$, significantly different from baseline.
An average blood pressure (BP) also measured over a 1-min period, and included measuring the systolic (SBP) and diastolic blood pressures (DBP) with an automatic digital BP meter (HEM-7020, OMRON, Tokyo, Japan). The subjects were requested to perform respiration at a rate of 10 times (exhalation for 3 seconds and inhalation for 3 seconds) per minute in rhythm with an electronic metronome (Digital metronome: DM-70, Seiko Watch Corporation, Tokyo, Japan)during the measurement so that the value evaluated as HF from the relationship between the frequency and respiration rate did not overlap LF [18]. Respiration training was conducted for 10 minutes under monitoring before each measurement and respiration was confirmed visually also during measurement, since the measurement was conducted under regulated respiration.

\subsection{Statistical Analysis}

The values for each parameter before SS (pre) were averaged over the 5-min period immediately before SS. One-way analysis of variance (ANOVA) using repeated measurements and two-way ANOVA were conducted for each numerical data set; Bonferroni's tests were used for post hoc analyses. SPSS, version 12.0 for Windows (SPSS, Chicago, IL, USA) was used for statistical analyses, and the statistically significant level was set at less than $5 \%$.

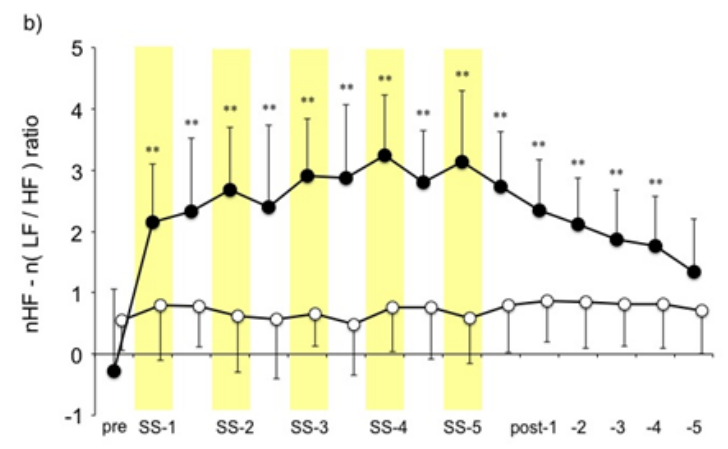

d)

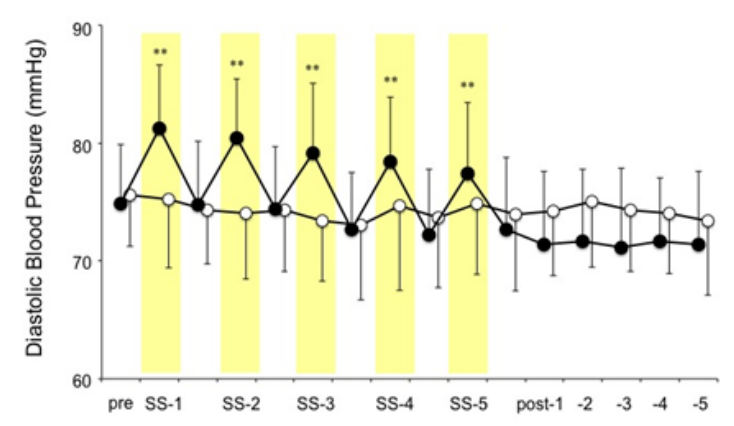




\section{Results}

The changes in each parameter are shown in Figure 2-a to e. The ROM increased significantly until 5-min post SS (Figure 2-a). The autonomic nerve activity significantly changed to parasympathetic dominance by SS as compared with pre. In addition, for SS, the autonomic nerve activity slowly changed to sympathetic dominance after completion of all sets of stretching, but these value did not return to pre during the 5-min after the completion of all sets of stretching, with parasympathetic dominance continuing by 4-min after SS(Figure 2-b). The values obtained for control showed no large change during measurement(Figure 2-b). No extrasystole was observed in any subjects by diagnosis with the analysis software for exclusive use. The changes in SBP, DBP and HR are shown in Figure 2-c, $d$ and e. These parameters transiently increased during SS and decreased after SS. In addition, HR significantly decreased after completion of all sets of SS (3-min).

\section{Discussion}

The major results of this study are as follows: 1 ) The balance of autonomic nerve activity shifts to a parasympathetic nerve-dominant state during passive SS; and2) the parasympathetic nerve-dominant state continues even after the completion of SS (for at least 5 minutes after completion). The study on the autonomic nerve activity upon passive SS in humans is valuable and the results of this study can be said to be a new fact which can be added to the findings concerning SS and relaxation.

Considering the precedent studies all together [5,6,7], when SS is conducted actively, the autonomic nerve activity shifts to a sympathetic nerve-dominant state during SS and a parasympathetic nerve-dominant state after the completion of SS. Also in this study, the balance of autonomic nerve activity shifts to a parasympathetic nerve-dominant state after SS, which supports the results of the precedent studies. However, unlike active SS, the response during SS shifted to a parasympathetic nervedominant state. This result indicates the possibility that the process differs between active SS and passive SS, although the response after the completion of SS is similar for both. Murata et al. [19] investigated autonomic nerve activity following passive SS in decerebrate cats as a precedent study of an animal experiment level. According to Murata et al. [19], cardiac sympathetic nerve activity increased only at the time of start of SS (where analysis was carried out in the condition in which parts of the cardiac vagal nerve and the stellate ganglion were cut), and this response has been confirmed muscle sympathetic nerve activity in human [20]. Since analysis was conducted for one minute in this study, not only cardiac sympathetic nerve activity that increases only at the start of SS, but also stimulation of the suppression system that subsequently occurs might be analyzed, and as a result, the balance of autonomic nerve activity is considered to shift to a parasympathetic nerve-dominant state. In addition,transient increases in SBP,DBP and HR have been reported in response to local SS of the triceps surae muscle [20,21,22], and our results support these previously described findings. Although the SS method was different in the previous studies, the transient changes in hemodynamic properties can be associated with mechanical stress and modulations of the baroreflex sensitivity and vagal tone during SS [20,21,22]. It is difficult to identify the mechanism from the results of this study; however, it is considered that the reduction in HR due to SS is mainly due to suppression of sympathetic nerve activity [2], and the reduction in HR plays a role in continuation of a parasympathetic nerve-dominant state by SS.

Limb position is considered to be one of the causes for the difference in autonomic nerve activity between active SS and passive SS. There are a large number of muscle spindles and proprioceptors in the diaphragm and the intercostals, which involve in up-and-down movements of the ribs [23]. In the trunk flexion which was conducted in the precedent studies, SS was conducted with the hip joint bent maximally so that a large pressure was applied to the thoracoabdominal part and muscle extension stimuli would occur at the muscles other than the hamstring muscle (for example, external oblique muscle and internal oblique muscle). As mentioned above, the influence of SS on the nervous system is known to be transmitted to the sites to which SS is not conducted $[9,10]$. Further, it is assumed that respiratory load (load compensation reflex) due to the position at which the trunk was anteverted might prevent respiration by an ordinary respiration method (with relaxation) to affect the balance of autonomic nerve activity.

There are two main limitations associated with the present study. The first limitaionsis that HRV analysis using SDPTG conducted. We used SDPTG to minimize invasion that included attachment of electrodes as much as possible. The usability of investigation of autonomic nerve activity using SDPTG has already been evidenced by Yamaguchi, et al.; however, influence of changes in body motion during SS, that is, due to dorsiflexion of the ankle joint, on measurements is unclear. This point should be sufficiently considered in further studies.The second limitation is that all the parameters were calculated as an average over 1-min intervals. According to Cui et al. [20], the HR increased between one and three beats during SS. This suggests that a hyper-acute effect of SS may occur, and future studies should employ a better temporal analysis, with times up to $1 \mathrm{~min}$. However, because Cui et al. [20] employed a different SS paradigm (5-s $\times 25$ sets), there is also a possibility that the SS performance time had an effect. The precise effect of SS time should be investigated in future studies.

In summary, autonomic nerve activity shifts to a parasympathetic nerve-dominant state by passive static stretching, and the effect continues for at least five minutes after the completion. The possibility that the response during SS may differ from the response during active static stretching is shown.

\section{References}

[1] American College of Sports Medicine Position Stand., "The recommended quantity and quality of exercise for developing and maintaining cardiorespiratory and muscular fitness, and flexibility in healthy adults,” Med Sci Sports Exerc, 30. 975-991. 1998. 
[2] Alter, M.J., "Science of flexibility," 3rd edition, Human Kinetics Pub, Champaign, Illinois, 2004.

[3] Khattab, K., Khattab, A.A., Ortak, J., Richardt, G., Bonnemeier, H., "Iyengar yoga increases cardiac parasympathetic nervous modulation among healty yoga practitioners,” Evid Based Complement Alternat Med, 4. 511-517. 2007.

[4] Lu, W.A., Kuo, C.D., "The effect of Tai Chi Chuan on the autonomic nervous modulation in older persons," Med Sci Sport Exerc, 35. 1972-1976. 2003.

[5] Saito, T., Hono, T., Miyachi, M., "Effects of stretching on cerebrocortical and autonomic nervous system activities and systemic circulation,” J Phys Med, 12. 2-9. 2001.

[6] Farinatti, P.T., Brandao, C., Soares, P.P., Duarte, A.F., "Acute effects of stretching exercise on the heart rate variability in subjects with low flexibility levels," J Strength Cond Res, 25. 1579-1585. 2011.

[7] Mueck-Weymann, M., Janshoff, G., Mueck, H., "Stretching increases heart rate variability in healthy athletes complaining about limited muscular flexibility," ClinAuton Res, 14. 15-18. 2004.

[8] Mohr, K.J., Pink, M.M., Elsner, C., Kvitne, R.S., "Electromyographic investigation of stretching: The effect of warm-up,” Clin J Sport Med, 8. 215-220. 1998.

[9] Avela. J., Kyrolainen, H., Komi, P.V.,"Altered reflex sensitivity after repeated and prolonged passive muscle stretching,” $J$ ApplPhysiol, 86. 1283-1291. 1999.

[10] Cramer, J.T., Housh, T.J., Weir, J.P., Johnson, G.O., Coburn, J.W., Beck, T.W., "The acute effects of static stretching on peak torque, mean power output, electromyography, and mechanomyography," Eur J ApplPhysiol, 93. 530-539. 2005.

[11] Mizuno, T., Matsumoto, M., Umemura, Y., "Viscoelasticity of the muscle-tendon unit is returned more rapidly than range of motion after stretching," Scnad J Med Sci Sports, 23. 23-30. 2013.

[12] Morse, C.I., Degans, H., Seynnes, O.R., Maganaris, C.N., Jones, D.A., "The acute effect of stretching on the passive stiffness of the human gastrocnemius muscle tendon unit," J Phyiol, 586. 97-106. 2008.

[13] Inami, T., Shimizu, T., Miyagawa, H., Inoue, M., Nakagawa, T., Takayanagi, F. Niwa, S.,"Effect of two passive stretching methods for triceps surae on dorsiflexion of ankle joint," J Phys Fitness Sports Med, 59. 549-554. 2010.

[14] Kawakami, Y., Oda, T., Kurihara, T., Chino, K., Nagayoshi, T. Kanehara, H., Fukunaga, T., Kuno, S., "Musculoskeletal factors influencing ankle joint range of motion in the middle-aged and elderly individuals,” Jpn J Phys Fitness Sports Med, 52. 149-156. 2003.

[15] Yamaguchi, K., Sasabe, T., Tajima, S., Watanebe, Y., "The evaluation of fatigue by acceleration plethysmogram,” J ClinExp Med, 22. 646-653. 1009.

[16] Task force of the European Society of Cardiology and the North American Society of Pacing and Electrophysiology, Heart rate variability. Standards of measurement, physiological interpretation, and clinical use. Circulation. 93. 1043-1065. 1996.

[17] Yoshida, Y., Yokoyama, K., Takada, H., Iwase, S.,"Heart rate variability before fainting under the graded local of artificial gravity," AutonNervSyst,43. 453-459. 2006.

[18] Matsumoto, T., Matsunaga, A., Hara, M., Saito, M., Yonezawa, R. Ishii, A., Kutsuna, T., Yamamoto, K., Masuda, T., "Effect of the breathing mode characterized by prolonged expiration on respiratory and cardiovascular responses and autonomic nervous activity during the exercise," Jpn J Phys Fit Sport Med, 57. 315326. 2008.

[19] Murata, J., Matsukawa, K., "Cardiac vagal and sympathetic efferent discharge are differentially modified by stretch of skeletal muscle," Am J Phyiol Heart Circ, 280. H237-H245. 2001.

[20] Cui, J., Blaha, C., Moradkhan, R., Gray, K.S., Sinoway, L. I., "Muscle sympathetic nerve activity responses to dynamic passive muscle stretch in humans," J Physiol, 576. 625-634. 2006.

[21] Drew, R.C., Bell, M.P.D., White, M.J., "Modulation of spontaneous baroreflex control of heart rate and indexes of vagal tone by passive calf muscle stretch during graded metaboreflex activation in humans,” J ApplPhysiol, 104. 716-723. 2008.

[22] Fisher, J.P., Bell, M.P.D., White, M.J., "Cardiovascular responses to human calf muscle stretch during varying levels of muscle metaboreflex activation," ExpPhysiol, 90. 773-781. 2005.

[23] Euler, C.V., "On the role of proprioceptors in perception and execution of motor acts with special reference to breathing," In: PengellyLD, Rebuch AS, Campbell EJM eds, Loaded breathing, Longman Canada, 139-154. 1973. 